\title{
Diseño e implementación de una rotación clínica a distancia para no-radioterapeutas en un servicio de radioterapia en un centro universitario durante la pandemia COVID-19
}

\section{Design and implementation of non presencial clinical rotation for non radiation oncologist in an academic radiotherapy service during COVID-19 pandemic}

\author{
Andrés Rosa Valencia ${ }^{1^{*}}$, Hernán Ramírez Skinner ${ }^{2}$, Tomás Merino ${ }^{3}$ \\ 1 , Departamento de Cirugía Oncológica y Maxilofacial, Pontificia Universidad Católica de Chile. \\ Airosa@uc.cl, https:/ / orcid.org/0000-0002-0962-6830 \\ 2, Departamento de Cirugía Oncológica y Maxilofacial, Pontificia Universidad Católica de Chile. \\ Hramirez.skinner@gmail.com \\ 3, Departamento de Hemato-Oncología, Pontificia Universidad Católica de Chile. \\ Tomasmerinolara@gmail.com \\ *Correspondencia: Andrés Ignacio Rosa Valencia, airosa@uc.cl \\ Recibido: 14-1-21; Aceptado: 22-4-21; Publicado: 29-4-21
}

Resumen: Introducción: La pandemia COVID-19 ha presentado desafíos en la educación de residentes que rotan por servicios de radioterapia. El objetivo de este trabajo es presentar una experiencia de diseño e implementación de una rotación a distancia en un servicio de radioterapia de un centro universitario. Métodos: Se utilizó el modelo de seis pasos de Kern para describir el diseño e implementación de una rotación a distancia. Resultados: Se presenta la experiencia en base a identificación del problema y evaluación general de necesidades, evaluación de necesidades específicas del estudiante y ambiente educativo, definición del propósito y objetivos de aprendizaje, selección de metodologías y estrategias educativas, implementación y evaluación de la experiencia. Conclusiones: Es posible desarrollar una rotación a distancia en un servicio de radioterapia durante la pandemia COVID-19.

Palabras clave: Radioterapia, rotación a distancia, tele-rotación, educación, residentes, COVID-19

\begin{abstract}
Introduction: COVID-19 pandemic has presented challenges in medical education of residents visiting radio oncology services. The aim of this study is present an experience of design and implementation of a non presential clinical rotation in an academic radiotherapy service. Methods: A Kern's six steps aproach will be used. Results: It's presented an experience based in identification of problems and general assesment of needs, evaluation of specífics needs of studient and educative enviroment, propouse definition and learning objetives, selection of metodologies and educative strategies, implementation and experience evaluation. Conclutions: Is posible to develop non presential rotation in a radiotherapy service during COVID-19 pandemic.
\end{abstract}

Keywords: Radiotherapy, non presential rotation, tele-rotation, education, residents, COVID-19 


\section{Introducción}

La pandemia COVID-19 ha planteado importantes desafíos a la educación médica de residentes para el presente y el futuro. Debido a la estrategia de salud pública para reducir la exposición evitando reuniones, disminuyendo el número de personas por superficie y limitando actividades presenciales, se ha hecho necesario replantear el funcionamiento de las unidades docente-asistenciales, incluyendo los servicios de radioterapia (1-3).

Las rotaciones clínicas en los servicios de radioterapia son un componente esencial en la educación oncológica de los residentes de diversas especialidades médicas, incluyendo Cirugía Oncológica, Cirugía Maxilofacial, Oncología Médica, Urología, Cuidados Paliativos y Ginecología Oncológica (4).

El objetivo de este trabajo es presentar la experiencia de diseño e implementación de una rotación clínica a distancia para no-radioterapeutas en el Servicio de Radioterapia de la Universidad Católica de Chile, tomando como ejemplo una rotación de los residentes del programa de especialización en Cirugía Oral y Maxilofacial.

\section{Métodos}

Se utilizó el modelo de seis pasos propuesto por Kern et al. (5-6) para describir en cada etapa el plan de diseño e implementación de la rotación clínica a distancia.

\section{Paso 1: Identificación del problema y evaluación general de necesidades}

Se realizó una identificación general del contexto y la necesidad de implementar la rotación a distancia para residentes de especialidades médicas, de acuerdo con la solicitud directa de aquellas especialidades.

\section{Paso 2: Evaluación de necesidades específicas de estudiantes y ambiente educativo}

Se concretó una entrevista telefónica o videoconferencia con el residente como primer acercamiento. En esta instancia se exploró el contexto del residente; si el motivo de su rotación forma parte del programa formal de la especialidad o es una rotación electiva, la duración de la rotación, motivaciones y experiencia previa. Se indagó la dedicación parcial o exclusiva a la rotación, su contexto social y familiar, la disponibilidad de espacio físico protegido para el teletrabajo, el acceso a internet en el lugar o la necesidad de movilización. Esta información permitió estimar el número de horas semanales que podría dedicar al trabajo en la rotación y la anticipación con la que debió conocer las actividades.

\section{Paso 3: Definición del propósito y objetivos de aprendizaje}

Se ajustaron los objetivos de aprendizaje de la rotación para adecuarse a la modalidad a distancia. Si los objetivos de aprendizaje de la rotación estaban previamente determinados en los programas propios de cada especialidad del pasante, se solicitaron estos objetivos. Si los objetivos de aprendizaje no estaban previamente determinados, se construyeron los objetivos para la rotación y se analizaron de acuerdo a la posibilidad de cumplimiento. Para esta construcción se utilizó la Taxonomía de Bloom adecuando cada objetivo de aprendizaje al contexto no presencial (7).

\section{Paso 4: Selección de metodologías y estrategias educativas}

Para cada objetivo de aprendizaje se estableció una actividad asociada. Si alguno de los objetivos de aprendizaje previamente establecidos no pudieran ser cumplidos mediante la rotación a distancia, se diseñó y planificó para ese caso una actividad presencial específica 
que permitiera el logro de ese objetivo. Posteriormente se construyó un instrumento específico que midió el logro de tal objetivo. Se estableció un sistema de evaluación o autoevaluación mediante una rúbrica explícita para asegurar el estándar mínimo para el instrumento. Se planificó al menos un espacio de retroalimentación en forma de una instancia virtual sincrónica.

\section{Paso 5: Implementación}

Las actividades que permitieran el logro de los objetivos de aprendizaje se llevaron a cabo durante el período de la rotación establecido. Los recursos e instancias se encontraron disponibles en formato online, sincrónico o asincrónico de acuerdo a la planificación. Las tutorías fueron individuales y las actividades de telemedicina contaron con la autorización expresa de los pacientes.

\section{Paso 6: Evaluación de la experiencia}

La evaluación de la experiencia, principal mecanismo para conocer si se lograron los objetivos de la rotación, contó con mecanismos sincrónicos para explorar los aspectos operacionales de la rotación y con una herramienta cuantitativa, que permitió sistematizar los resultados y plantear mejoras.

\section{Resultados}

El contexto COVID-19 ha planteado la necesidad de disminuir la posibilidad de contagio limitando las reuniones, disminuyendo el número de personas por superficie y limitando actividades presenciales en los programas de formación, incluyendo las rotaciones clínicas de los programas de especialidades médicas por el Servicio de Radioterapia de la Universidad Católica de Chile. En este contexto, el programa de especialidad en Cirugía Oral y Maxilofacial solicitó una rotación por este servicio para uno de sus residentes, planteando la posibilidad de que esta rotación fuera a distancia.

En este caso se solicitó una rotación como parte del programa de especialidad, de un mes de duración, con dedicación exclusiva, sin experiencia previa, siendo dictado por la misma institución. Las condiciones tecnológicas del residente fueron adecuadas para realizar la rotación virtual, sin necesidad de movilización.

Los objetivos de la rotación se encontraban previamente establecidos, declarados en el programa de Cirugía Oral y Maxilofacial y se estableció que aquellos eran susceptibles de cumplir en la rotación no presencial. Así, los objetivos eran:

1) Describir las bases teóricas que fundamentan la radioterapia

2) Describir la técnica de radioterapia de cabeza y cuello

3) Describir las complicaciones de la radioterapia de cabeza y cuello

- Mucositis

- Xerostomía

- $\quad$ Osteoradionecrosis

4) Comprender el rol de la radioterapia en el modelo de tratamiento del paciente con cáncer

Para lograrlos, se estableció un tipo de actividad que permitiera el logro de cada objetivo. Así, para el objetivo 1, 2 y 3, se realziaron lecturas complementarias, palabras 
clave y bibliografía. Para el objetivo 4, participación en comités a distancia en tiempo real de casos reales.

Para medir el logro de los objetivos 1 a 4, se planificó con el pasante la realización de un portafolio de tipo evaluación formativa incluyendo:

- Generalidades de los temas contenidos en los objetivos 1 a 3 (midió logro de objetivo $1,2$ y 3$)$.

- Reflexión escrita (midió logro del objetivo 4).

- Autoevaluación formativa del portafolio.

- Feedback oral o escrito del tutor (staff) encargado.

Para evaluar formativamente el portafolio, se utilizó la rúbrica de autoevaluación disponible en la Tabla 1. El estándar de la rotación es logrado en todos los ítems. Se realizó una instancia final de retroalimentación sincrónica.

Tabla 1: Rúbrica de autoevaluación

\begin{tabular}{|l|l|l|l|}
\hline & \multicolumn{2}{|c|}{ Logrado Parcialmente logrado } & No logrado \\
\hline Objetivos & $\begin{array}{l}\text { El portafolio incluye } \\
\text { todos los temas } \\
\text { declarados en los } \\
\text { objetivos y yon } \\
\text { desarrollados de forma } \\
\text { clara. }\end{array}$ & $\begin{array}{l}\text { El portafolio incluye todos los } \\
\text { temas declarados en los } \\
\text { objetivos, pero no se } \\
\text { desarrolla de forma clara. }\end{array}$ & $\begin{array}{l}\text { El portafolio } \\
\text { incluye todos los } \\
\text { temas declarados en } \\
\text { los objetivos } \\
\text { incluye conceptos } \\
\text { errados. }\end{array}$ \\
\hline Bibliografía & $\begin{array}{l}\text { Incluye bibliografía. La } \\
\text { bibliografía es relevante } \\
\text { y actualizada. }\end{array}$ & $\begin{array}{l}\text { Incluye bibliografía pero no es } \\
\text { relevante o actualizada. }\end{array}$ & $\begin{array}{l}\text { No } \\
\text { bibliografía. }\end{array}$ \\
\hline Reflexión & $\begin{array}{l}\text { El portafolio incluye una } \\
\text { reflexión personal en } \\
\text { torno al tema. Esta } \\
\text { relaciona la bibliografía } \\
\text { relevante con la rotación } \\
\text { realizada. }\end{array}$ & $\begin{array}{l}\text { El portafolio incluye una } \\
\text { reflexión personal en torno al } \\
\text { tema que no se relaciona con } \\
\text { la rotación y/o no incluye } \\
\text { bibliografía. }\end{array}$ & $\begin{array}{l}\text { No incluye reflexión } \\
\text { personal. }\end{array}$ \\
\hline
\end{tabular}

Para cada objetivo de aprendizaje se estableció una actividad asociada en la rotación, organizada en la Tabla 2.

Tabla 2: Actividades y objetivos de aprendizaje

\begin{tabular}{|ll|l|}
\hline \multicolumn{1}{|c|}{ Actividades } & \multicolumn{1}{c|}{ Objetivos de aprendizaje } \\
\hline $\begin{array}{l}\text { Clase introductoria a radioterapia } \\
\text { Recurso online asincrónico: } \\
\text { https://voices.uchicago.edu/roecsg/intr } \\
\text { oduction-to-radiation-oncology/ }\end{array}$ & $\begin{array}{l}\text { Describir las bases teóricas que fundamentan la } \\
\text { radioterapia }\end{array}$ \\
\hline $\begin{array}{l}\text { Comité de Radioterapia } \\
\text { Chart de Radioterapia } \\
\text { - Seminario introductorio a la técnica de }\end{array}$ & $\begin{array}{l}\text { Describir la técnica de radioterapia de cabeza y } \\
\text { cuello }\end{array}$ \\
\hline
\end{tabular}




\begin{tabular}{|c|c|}
\hline 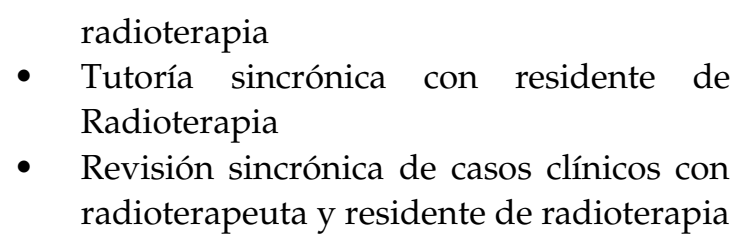 & \\
\hline $\begin{array}{l}\text { - Comité de Cabeza y Cuello } \\
\text { - Clase asincrónica Radioterapia de Cabeza } \\
\text { - } \quad \text { Consullo para odontología } \\
\text { - Con Telemedicina }\end{array}$ & $\begin{array}{l}\text { Describir las complicaciones de la radioterapia } \\
\text { de cabeza y cuello }\end{array}$ \\
\hline $\begin{array}{l}\text { - Comité de Radioterapia } \\
\text { - Comité de Cabeza y Cuello } \\
\text { - Comité de Mama } \\
\text { - Comité de Melanoma } \\
\text { - Tutoría sincrónica con radioterapeuta } \\
\text { - Consultas por Telemedicina }\end{array}$ & $\begin{array}{l}\text { Comprender el rol de la radioterapia en el } \\
\text { modelo de tratamiento del paciente con cáncer }\end{array}$ \\
\hline
\end{tabular}

Para la evaluación de la experiencia se realizó una encuesta final de percepción abierta respecto a la rotación y la evaluación por mecanismos habituales institucionales.

\section{Discusión.}

Las rotaciones clínicas en los programas de especialidades han tenido que realizarse ajustadas a la realidad del COVID-19 (8-11). En este servicio de radioterapia se planteó la posibilidad de realizar rotaciones virtuales para no-radioterapeutas y de acuerdo a la experiencia en el caso de estudio, parece ser una opción viable principalmente debido a la disponibilidad de herramientas de telecomunicación, tecnología y virtualización de las reuniones habituales (10). Para asegurar un óptimo desarrollo de esta experiencia, este caso evidenció que la caracterización del residente es un hito fundamental, directamente implicado en la planificación de la experiencia de rotación a distancia.

Los objetivos de aprendizaje deben ser cuidadosamente construidos para que estén en concordancia con las actividades virtuales posibles de realizar. Trabajar estos objetivos permite identificar aquellos que no son posibles cumplir a distancia o que deben ser abordados mediante actividades personalizadas (9).

La evaluación online supone un desafío y ha sido necesario usar sistemas de evaluación continua, individualizada y centrada en la construcción de planes individuales, en especial en la rotación de residentes de diversas especialidades, que constituyen cada uno un contexto educativo con necesidades particulares (10). En este caso, la retroalimentación sincrónica durante una rotación a distancia fue un hito fundamental para detectar precozmente posibles obstáculos en el desarrollo de la misma. A su vez, la evaluación final permitió sistematizar la información y construir planes de mejora.

Si bien fue posible cumplir con los objetivos de aprendizaje propuestos, aún no están claras las consecuencias de reemplazar la experiencia de una rotación presencial, principalmente debido a la ausencia de factores relacionados con el intercambio físico, socialización cultural y contexto formativo que otorga la presencialidad (11). 


\section{Limitaciones del estudio}

Este estudio corresponde al reporte de un caso y por lo tanto es afecto de sesgo. Es necesario agrupar mas experiencias de residentes de esta $\mathrm{u}$ otras especialidades para poder obtener resultados reproducibles que permitan obtener conclusiones claras.

\section{Conclusión}

- Mediante el abordaje curricular sistemático, es posible diseñar e implementar una rotación a distancia para no radioterapeutas en un servicio de radioterapia, permitiendo mantener la formación de residentes en el periodo COVID-19.

Financiación: No ha habido financiación.

Declaración de conflicto of interés: Los autores declaran no tener ningún conflicto de intereses.

\section{Referencias}

1. Al-Balas M, Al-Balas H I, Jaber H M et al. Distance learning in clinical medical education amid COVID-19 pandemic in Jordan: current situation, challenges, and perspectives. BMC medical education 2020; 20(1): 1-7. https://doi.org/10.1186/s12909-020-02257-4

2. Hilburg R, Patel N, Ambruso S et al. Medical Education During the Coronavirus Disease-2019 Pandemic: Learning From a Distance. Adv Chronic Kidney Dis. 2020; 27(5): 412-417. https://doi.org/10.1053/j.ackd.2020.05.017

3. Lucey CR, Johnston SC. The Transformational Effects of COVID-19 on Medical Education. JAMA. 2020; 324(11):1 033-1034. https://doi.org/10.1001/jama.2020.14136

4. Dooley S, Sim AJ, Campbell SR, Jeans EB, Anderson JD, Tye K, Goodman CR. Opportunities to Improve Radiation Oncology Medical Education in the Post-Pandemic Era. Int J Radiat Oncol Biol Phys. 2020; 108(2): 455-457. https://doi.org/10.1016/j.ijrobp.2020.06.038

5. Thomas P A, Kern D E, Hughes MT, Chen BY. (Eds.) (2016). Curriculum development for medical education: a six-step approach. JHU Press. https://jhu.pure.elsevier.com/en/publications/curriculumdevelopment-for-medical-education-a-six-step-approach

6. Robertson AC, Fowler LC, Niconchuk J, Kreger M, Rickerson E, Sadovnikoff N, Hepner DL, Bader AM, Mcevoy MD, Urman RD. Application of Kern's 6-Step Approach in the Development of a Novel Anesthesiology Curriculum for Perioperative Code Status and Goals of Care Discussions. J Educ Perioper Med. 2019; 21(1): E634. https://pubmed.ncbi.nlm.nih.gov/31406705/

7. ¿Sabes en qué consiste la taxonomía de Bloom? En Universia.net. Visitado 2122 de abril de 2021, en https://www.universia.net/cl/actualidad/orientacion-academica/sabes-que-consiste-taxonomia-bloom1163730.html

8. Gottlieb M, Landry A, Egan DJ, Shappell E, Bailitz J, Horowitz R, Fix M. Rethinking Residency Conferences in the Era of COVID-19. AEM Educ Train. 2020; 4(3): 313-317. https://doi.org/10.1002/aet2.10449

9. Rivera S, Rosenabaum A, Rojas P, et al. Contacto clínico precoz: experiencia de diseño e implementación de un curso de servicio comunitario en medicina. Educación Médica 2019; 20: 79-85. https://doi.org/10.1016/j.edumed.2018.03.022

10. García-Peñalvo FJ, Abella-García V, Corell A, Grande M. La evaluación online en la educación superior en tiempos de la COVID-19. Education in the knowledge society (EKS) 2020; 21(4): 1-26. https://doi.org/10.14201/eks.23013

11. Almazón A. Covid-19: ¿Punto Sin Retorno de la Digitalización de la Educación?. Revista Internacional de Educación para la Justicia Social, 2020; 9(3). https://revistas.uam.es/riejs/article/view/12089/12009 